\title{
Polibotánica
}

ISSN electrónico: 2395-9525

POLIBETÁNICA polibotanica@gmail.com Instituto Politécnico Nacional México http:www.polibotanica.mx

\section{HETEROGENEIDAD ESTRUCTURAL DEL MANGLAR COMO RESPUESTA A FACTORES AMBIENTALES Y ANTRÓPICOS EN EL SOCONUSCO, CHIAPAS, MÉXICO.}

\section{STRUCTURAL HETEROGENEITY OF MANGROVES AS A RESPONSE OF ENVIRONMENTAL AND ANTHROPIC FACTORS IN SOCONUSCO, CHIAPAS, MEXICO.}

Romero-Berny, E. I.; C. Tovilla-Hernández, N. Torrescano-Valle, y B. Schmook

HETEROGENEIDAD ESTRUCTURAL DEL MANGLAR COMO RESPUESTA A FACTORES AMBIENTALES Y ANTRÓPICOS EN EL SOCONUSCO, CHIAPAS, MÉXICO. STRUCTURAL HETEROGENEITY OF MANGROVES AS A RESPONSE OF ENVIRONMENTAL AND ANTHROPIC FACTORS IN SOCONUSCO, CHIAPAS, MEXICO.

POLIBETÁNICA Instituto Politécnico Nacional
Núm. 47: 39-58 México. Enero 2019

DOI: $10.18387 /$ polibotanica.47.4 


\section{HETEROGENEIDAD ESTRUCTURAL DEL MANGLAR COMO RESPUESTA A FACTORES AMBIENTALES Y ANTRÓPICOS EN EL SOCONUSCO, CHIAPAS, MÉXICO.}

\section{STRUCTURAL HETEROGENEITY OF MANGROVES AS A RESPONSE OF ENVIRONMENTAL AND ANTHROPIC FACTORS IN SOCONUSCO, CHIAPAS, MEXICO.}

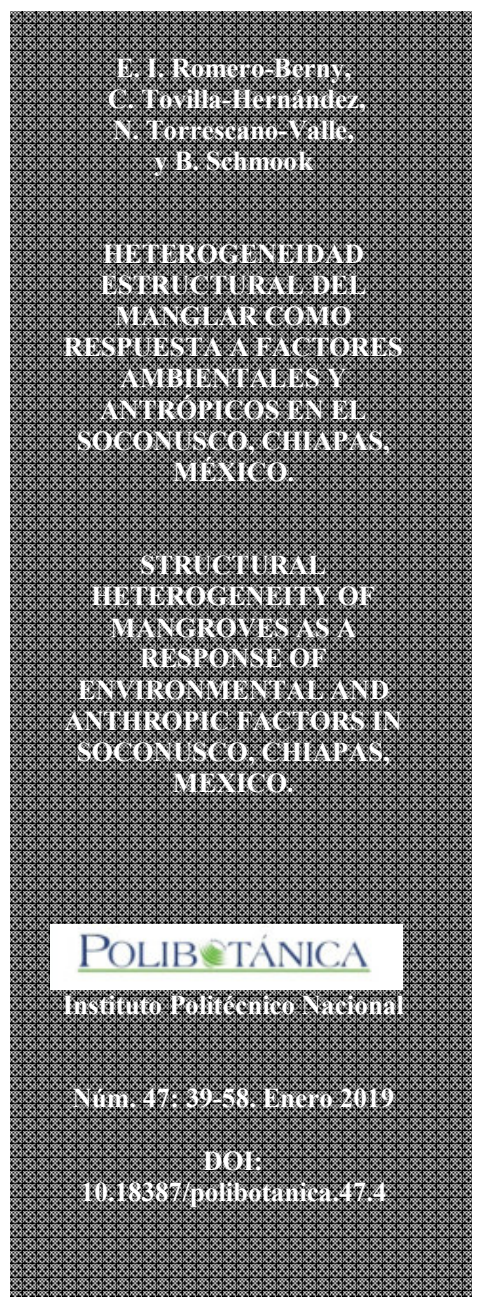

\author{
E. I. Romero-Berny/ emilio.romero.ex@unicach.mx \\ Centro de Investigaciones Costeras, Instituto de Ciencias Biológicas, \\ Universidad de Ciencias y Artes de Chiapas. J.J. Calzada y Calzada de Guadalupe,
} Col. Evolución, CP 30500, Tonalá, Chiapas, México.

\section{Tovilla-Hernández}

Grupo Manejo de Cuencas y Zonas costeras, Departamento de Ciencias de la Sustentabilidad, El Colegio de la Frontera Sur-Unidad Tapachula. Carretera a Antiguo Aeropuerto km 2.5, CP 30700, Tapachula, Chiapas, México.

\author{
N. Torrescano-Valle \\ B. Schmook \\ Grupo Diversidad y Dinámica de Ecosistemas del Sureste de México, \\ Departamento de Conservación de la Biodiversidad, \\ El Colegio de la Frontera Sur-Unidad Chetumal. \\ Av. Centenario km 5.5, CP 77014, Chetumal, Quintana Roo, México.
}

RESUMEN: Se presenta una descripción de la heterogeneidad estructural de los manglares en la región del Soconusco, Chiapas, México. Su estructura fue analizada considerando un inventario forestal realizado en 40 unidades de muestreo de $300 \mathrm{~m}^{2}$. La densidad de árboles vivos y muertos en pie, tocones y plántulas fue estimada por hectárea. Las especies más dominantes de acuerdo al índice de Valor de Importancia fueron Rhizophora mangle (29.68\%) y Laguncularia racemosa (27.33\%). La estructura general de los manglares en el Soconusco, en términos de su densidad ( 2792 fustes ha ${ }^{-1}$ ), área basal $\left(36.5 \mathrm{~m}^{2} \mathrm{ha}^{-1}\right)$ y altura $(15.5 \mathrm{~m})$ es mayor a la registrada en otras áreas del Pacífico mexicano. El $86.3 \%$ de los árboles muestreados presentan diámetro a la altura del pecho $\leq 17.7 \mathrm{~cm}$. La clasificación de la vegetación, basada en el Valor de Importancia relativa de las especies, definió cinco grupos de manglar. Destaca un grupo dominado por Pachira aquatica. La más alta densidad de árboles vivos y tocones fue registrada en grupos mixtos de Conocarpus erectus, mientras la mayor densidad de plántulas se registró en grupos monoespecíficos de Avicennia germinans. La composición de las comunidades varió significativamente asociada a las condiciones ambientales, y fueron definidas en su estructura por el área basal, diámetro de copa, altura y densidad, así como por factores que pueden asociarse con una extracción selectiva de árboles, como las densidades de tocones y plántulas. Se recomienda considerar las características estructurales de mayor importancia (altura, área basal, cobertura, densidad de tocones) para las agrupaciones de manglar y sus conductores en la planeación de su manejo.

Palabras clave: Estructura de la vegetación, Valor de importancia, características ambientales, grupos de manglar, Pacífico mexicano.

ABSTRACT: Structural characteristics and dominance patterns in mangroves have been linked to environmental, anthropogenic and ecophysiological factors. The Soconusco on the southern coast of the Mexican Pacific is an environmentally heterogeneous region, with high availability of freshwater and different degrees of 
impact. The goal of this study was to analyze the mangrove groups, including their structure and dominance pattern under the influence of eight environmental factors (salinity, flood level, $\mathrm{pH}$, air temperature, soil temperature, relative humidity, and content of organic matter $0-30 \mathrm{~cm}$, 0-60 cm). The characteristic physical features and caused by the impact were described for each group. Mangrove structure was evaluated considering a forest inventory conducted in 40 sampling units $\left(300 \mathrm{~m}^{2}\right)$. Density of live and standing dead trees, stumps and seedlings was estimated for 1ha. The dominance for each species was determined using the relative Importance Value. The identification of groups was carried out with ordination and classification routines. The species with the highest Importance Values were Rhizophora mangle (29.68\%) and Laguncularia racemosa (27.33\%). Comparatively, the general structure of mangroves in Soconusco in terms of density (2 792 steams ha $\left.\mathrm{h}^{-1}\right)$, basal area $\left(36.5 \mathrm{~m}^{2} \mathrm{ha}^{-1}\right)$ and height $(15.5 \mathrm{~m})$ is greater than in other areas of the Mexican Pacific coast. Classification of vegetation data, based on the Relative Importance Value Index, defined five mangrove groups. Outstanding is the presence of a community dominated by Pachira aquatica. The highest density of trees and stumps was recorded in Conocarpus erectus grouping, while the highest density of seedlings was in Avicennia germinans grouping. $86.3 \%$ of sampled trees present $\leq$ $17.7 \mathrm{~cm}$ diameter at breast height. The variables that correlate significantly with the axis 2 of the ordination biplot were organic matter $(30-60 \mathrm{~cm})$, salinity and flood level. Canonical Discriminant Analysis corroborated the differences between mangrove groups, with the first two canonical axes explaining $89.05 \%$ of the variation in the data. The community composition varied associated with physical and environmental conditions, and were structurally defined by basal area, crown diameter, height and density, as well as by factors that can be associated with an anthropic impact such as stump and seedling densities. It is recommended to consider the characteristics of each mangrove group to plan their management.

Key words: Vegetation structure, Importance value, Environmental characteristics, Mangrove groups, Mexican Pacific.

\section{INTRODUCCIÓN}

Los manglares son comunidades vegetales halófitas, arbustivas o arbóreas, típicas de las zonas costeras tropicales y subtropicales. Estas plantas comparten adaptaciones morfológicas y fisiológicas que les permiten desarrollarse en suelos frecuentemente anaeróbicos, ricos en materia orgánica y con acentuada fluctuación hidroperiódica (Hogarth, 2007; Twilley, 1998).

La estructura de los manglares se integra por un conjunto de variables tales como la riqueza y dominancia de especies, densidad, altura y área basal, que definen los atributos ecológicos de la comunidad (Cintrón \& Schaeffer, 1984; Ramos-Durón, Quiróz-Flores, Ramírez García-Armora, \& Lot-Helgueras, 2004). Se ha demostrado que los patrones de heterogeneidad estructural en una comunidad de mangles se relacionan estrechamente con procesos geomorfológicos ocurridos durante el Holoceno (Domínguez-Cadena, Riosmena-Rodríguez, \& León-de la Luz, 2016; González-Zamorano, Lluch-Cota, \& Nava-Sánchez, 2013; Torrescano \& Islebe, 2006). Cada especie se adapta de forma diferente a las condiciones ambientales impuestas por la geomorfología, ocupando una zona fisiológicamente óptima en un gradiente ambiental, lo que a nivel de comunidad implica el desarrollo de características particulares de fisiografía, estructura y composición (López-Portillo \& Ezcurra, 1989; Lugo \& Snedaker, 1974; Méndez-Linares, López-Portillo, Hernández-Santana, Ortiz-Pérez, \& Oropeza-Orozco, 2007). Sin embargo, a pesar de contarse con una amplia línea base sobre la distribución espacial de los manglares, su relación con otras variables fisicoquímicas del suelo y agua aún ha sido poco evaluada a escalas locales.

Diversos estudios han evidenciado que los impactos naturales y antrópicos, tales como huracanes, la tala selectiva, cambios de uso de suelo o alteración de la hidrodinámica, pueden afectar la estructura del manglar en periodos de tiempo relativamente cortos (Agraz-Hernández \& Flores-Verdugo, 2014; Rodríguez-Zúñiga, Ramírez-García, \& Gutiérrez-Granados, 2011; 
Smith et al., 2009; Tovilla-Hernández, de la Lanza-Espino, \& Orihuela-Belmonte, 2001). Por lo tanto, la caracterización estructural de los manglares es un procedimiento indispensable en el monitoreo de estos ecosistemas. Esta información básica constituye parte de los protocolos de valoración del potencial de captura de carbono y para la rehabilitación y restauración de humedales costeros (Flores-Verdugo et al., 2007; Xiaonan, Xiaoke, Lu, \& Zhiyun, 2008; Zaldívar-Jiménez et al., 2010).

Las condiciones ambientales en la costa del Pacífico mexicano presentan un marcado gradiente climático, que varía de semiárido en el norte a húmedo en el sur (Flores-Verdugo, de la LanzaEspino, Contreras-Espinosa, \& Agraz-Hernández, 2001). Las diferencias locales en la duración temporal de las estaciones lluviosa y seca, cambios de temperatura y número de ríos a lo largo de la costa Pacífica (de la Lanza-Espino, Ortiz-Pérez, \& Carbajal-Pérez, 2013) han conducido a hipótesis que plantean cambios significativos en la estructura de las comunidades bióticas en función de las variaciones ambientales aún dentro de una misma región (Tapia-García, GarcíaAbad, Carranza-Edwards, \& Vázquez-Gutiérrez, 2007).

El estado de Chiapas, en el Pacífico sur mexicano, presenta una extensión de 46,804 ha de manglares (Troche-Souza et al., 2016). Al sureste del estado, en la región Soconusco, la amplitud de la planicie costera, mayores precipitaciones y abundante descarga fluvial, favorecen en los manglares un desarrollo estructural comparativamente mayor a los de otras regiones en el Pacífico mexicano (Flores-Verdugo et al., 2001). Son escasas las investigaciones realizadas sobre estructura de los manglares en Chiapas (Montes-Cartas, Castillo-Argüero, \& López-Portillo, 1999; Ramírez-García \& Segura-Zamorano, 1994; Tovilla-Hernández \& Romero-Berny, 2012), y pocas se han enfocado a determinar las variables que definen los patrones ecológicos para cada tipo de comunidad.

Dadas las evidencias que sustentan la delineación de tipos de comunidades con rasgos estructurales específicos a escalas locales (Duke, 2006; González-Zamorano et al., 2013; TrejoTorres, Durán, \& Olmsted, 1993) y la provisión diferenciada de servicios ecosistémicos (Ewel, Twilley, \& Ong, 1998), los objetivos de esta investigación fueron: i) analizar la estructura de las comunidades de manglar en el Soconusco a partir de una clasificación de enfoque fisiográfico y su respuesta a factores ambientales, y ii) determinar los rasgos físico-estructurales que caracterizan a cada grupo de manglar identificado en la clasificación.

\section{MATERIAL Y MÉTODOS}

\section{Área de estudio}

El área estudiada se localiza en la zona meridional de la región del Soconusco en el estado de Chiapas, costa del Golfo de Tehuantepec, Pacífico sur de México. Las comunidades de manglar se asocian al sistema lagunar estuarino de El Cabildo-Amatal-Gancho Murillo, con una extensión de 12872 ha, entre las coordenadas $14^{\circ} 44^{\prime} 30^{\prime \prime}$ y $14^{\circ} 31^{\prime} 58^{\prime \prime}$ de latitud norte, $92^{\circ}$ $25^{\prime} 50^{\prime \prime}$ y $92^{\circ} 11^{\prime} 18^{\prime \prime}$ de longitud oeste (Ovalle-Estrada \& Vásquez-Lule, 2009) (fig. 1). El clima de la región se caracteriza como cálido-subhúmedo con una precipitación anual de 1578 $\mathrm{mm}$ y temperatura media de $27^{\circ} \mathrm{C}$. Existe marcada estacionalidad climática con dos temporadas bien definidas: lluviosa que se extiende de mayo a noviembre, y seca de diciembre a abril (Gobierno de Chiapas, 2010). La vegetación de esta región incluye, además de manglares compuestos por cuatro especies típicas para el neotropico (Rhizophora mangle L., Avicennia germinans (L.) L., Laguncularia racemosa L. (Gaertn.), Conocarpus erectus L.), a selvas inundables de Pachira aquatica Aubl., tulares (Typha domingensis Pers.) y vegetación secundaria con componentes de selva subcaducifolia como Miconia argentea (Sw.) DC., Cynometra oaxacana Brandegee, Guazuma ulmifolia Lam. y Sabal mexicana Mart. (Breedlove, 1981; Pennington \& Sarukhán, 2005). El uso del suelo adyacente es principalmente agrícola con diversos cultivos tropicales, aunque recientemente se ha presentado un acelerado desarrollo en los sectores industrial y turístico que ha ocasionado pérdida y fragmentación de manglares 
(Fernández, 2004; Romero-Berny, Acosta-Velázquez, Tovilla-Hernández, Schmook, \& GómezOrtega, 2015).

La región posee relevancia biológica y es altamente vulnerable al impacto ambiental, debido a su intensa dinámica social y productiva. En esta región se encuentran áreas con las siguientes categorías de manejo y conservación: Región Marina Prioritaria Núm. 40, Región Hidrológica Prioritaria Núm. 32, Área de Importancia para la Conservación de Aves Núm. 51, sitios RAMSAR, sitio de manglar con relevancia biológica PS27 y Zonas Sujetas a Conservación Ecológica Cabildo-Amatal y El Gancho-Murillo (Gobierno de Chiapas, 2010; Ovalle-Estrada \& Vásquez-Lule, 2009).

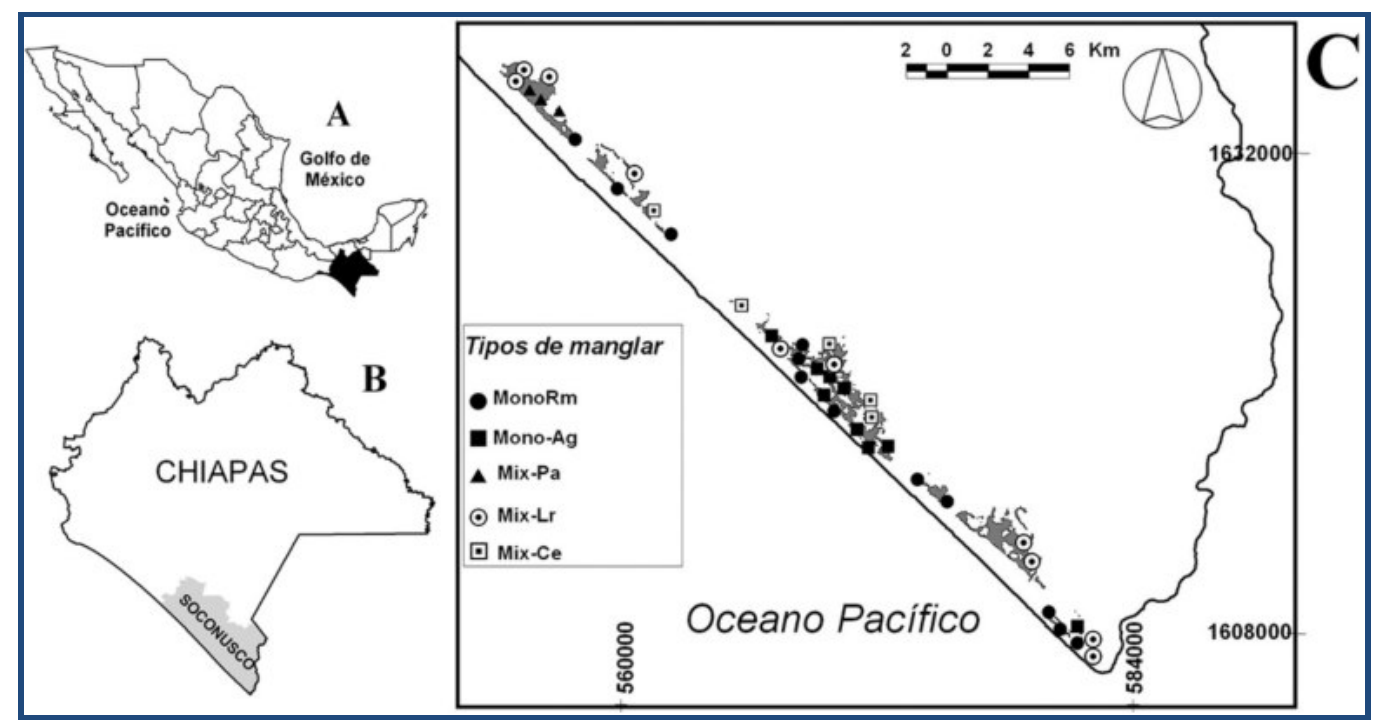

Fig. 1. Localización. A) México, B) Estado de Chiapas y región del Soconusco (sombreado), C) Sistema Cabildo Amatal-Gancho Murillo. Se muestran los tipos de comunidad de manglar: (Mono Rm) Monoespecífica de Rhizophora mangle, (Mono Ag) Monoespecífica de Avicennia germinans, (Mix Pa) Mixta de Pachira aquatica,

(Mix Lr) Mixta de Laguncularia racemosa, (Mix Ce) Mixta de Conocarpus erectus.

\section{Diseño de muestreo y caracterización estructural}

La estructura de la vegetación se determinó en 40 unidades de muestreo (UM) rectangulares de $300 \mathrm{~m}^{2}$, subdivididas en cuatro cuadrantes de 5 x $15 \mathrm{~m}$ (Valdéz-Hernández, 2002), y distribuidas de manera estratificada, considerando como criterios al tipo fisiográfico de manglar (cuenca o ribereño) (sensu Lugo \& Snedaker, 1974) y el grado de perturbación aparente (evidencia de tala). En cada UM se midió el diámetro a la altura del pecho (DAP) con una cinta diamétrica, registrándose únicamente el de aquellos fustes que presentaron DAP $\geq 2.5 \mathrm{~cm}$. Esta variable se midió del fuste a $1.5 \mathrm{~m}$ de altura con respecto al nivel del suelo, y para el caso de $R$. mangle, esta medida se tomó encima de la última raíz aérea. El diámetro de los tocones (diámetro a la altura del tocón, DAT) y árboles muertos también fue medido. La altura total y la cobertura foliar (diámetro mayor y diámetro menor de copa) fueron estimadas utilizando un hipsómetro láser (marca Haglöf). El número de plántulas fue registrado en cuatro subunidades de $1 \mathrm{~m}^{2}$ establecidas en los extremos de cada unidad. Se consideraron como plántulas a las plantas con DAP $<2.5 \mathrm{~cm}$ y menores a $1.3 \mathrm{~m}$ de altura, cuya densidad se consideró como un indicador de la regeneración potencial del manglar (Valle, Osorno-Arango, \& Gil-Agudelo, 2011). 
Los valores de densidad fueron estimados como el promedio de individuos \pm 1 error estándar (E.E) y desviación estándar (D.E.) en 1 ha. Se calculó el área basal (AB) por tallo con la siguiente fórmula (Cintrón \& Schaeffer, 1984; Pool, Snedaker, \& Lugo, 1977):

$$
A B=\pi\left(\frac{D A P}{2}\right)^{2}
$$

La cobertura foliar (CF) por árbol se calculó asumiendo la proyección de una copa elipsoidal sobre el suelo (Villacencio-García, Espinoza-Aréchiga, Hernández-Álvarez, GallegosRodríguez, \& Santiago-Pérez, 2009):

$$
C F=\pi\left[\frac{(\text { Diámetro mayor })(\text { Diámetro menor })}{2}\right]
$$

La cobertura relativa del dosel (CD) fue estimada con la siguiente fórmula (Zarco-Espinosa, Valdéz-Hernández, Ángeles-Pérez, \& Castillo-Acosta, 2010):

$$
C D=\left(\frac{C F \text { de todos los árboles }}{\text { Area de muestreo }}\right) 100
$$

Se calcularon los valores de densidad, frecuencia y dominancia de cada especie, y se estimó el Valor de Importancia Relativa (VIR) a partir de la suma de los valores relativos de esas variables (Pool et al., 1977; Ramos-Durón et al., 2004). Las comunidades se clasificaron de acuerdo con su fisiografía (cuenca, borde y ribereño) (Lugo \& Snedaker, 1974) y se describieron en función de sus parámetros estructurales promedio y composición, considerándose monoespecíficas cuando una sola especie presentó dominancia relativa $\geq 90 \%$ (Madrigal, 1994).

\section{Caracterización ambiental}

Al centro de cada UM se midieron los parámetros físico-ambientales en dos meses distintos correspondiendo a cada temporalidad (seca, febrero 2009; lluviosa, julio 2010; 80 mediciones). La salinidad intersticial (tomada entre $0-30 \mathrm{~cm}$ del suelo), $\mathrm{pH}$ y temperatura del suelo se midieron con un equipo multiparamétrico (YSI 55) y termómetro, así como el nivel de inundación con una regleta graduada. Por sitio se recolectaron dos muestras de suelo a profundidades de $0-30 \mathrm{~cm}$ y $30-60 \mathrm{~cm}$, las cuales fueron secadas a temperatura ambiente y tamizadas a un tamaño de partícula $<2 \mathrm{~mm}$. Para determinar el contenido porcentual de materia orgánica en las muestras tamizadas se utilizó el método de combustión seca $\left(375^{\circ} \mathrm{C}\right)$ y gravimetría (Ramos-Durón et al., 2004). La temperatura del aire y humedad relativa se midieron con un anemómetro portátil (marca Lutron), bajo el supuesto de que algunos factores de perturbación como la extracción selectiva, pueden conducir a cambios en estas variables dentro de un rodal (Tovilla-Hernández et al., 2001).

\section{Análisis de datos}

Se realizaron análisis de ordenación (escalamiento multidimensional-no métrico, NMDS por sus siglas en inglés) y clasificación por conglomerados (UPGMA) de los manglares sobre una matriz de Bray-Curtis para los datos de VIR transformados a raíz cuarta. La significancia de las diferencias estructurales entre cada grupo $(\alpha=0.05)$ se determinó con un Análisis de Similaridad de una vía (ANOSIM por sus siglas en inglés; 9999 permutaciones). La relación entre el gradiente espacial de la ordenación y los parámetros ambientales se exploró mediante coeficientes de Pearson. Estos análisis se realizaron utilizando el paquete PERMANOVA+ para PRIMER 6 (Anderson, Gorley, \& Clarke, 2008). 
Las variables de densidad, área basal, altura, cobertura de copa y cobertura de dosel de cada grupo de manglar identificado fueron comparadas por separado mediante un Análisis de Varianza de una vía (ANOVA por sus siglas en inglés), a excepción de los datos de densidad de tocones y árboles muertos en pie, los cuales fueron comparados con un análisis no paramétrico de Kruskall-Wallis, al no cumplirse el supuesto de normalidad y homogeneidad de varianzas (prueba de Shapiro-Wilks). La agrupación de medias se llevó a cabo mediante pruebas post hoc de diferencias honestamente significativas (Tukey) y de comparación múltiple no paramétrica a niveles de significancia del 5\% (Sokal \& Rohlf, 1994). Las variables físico-estructurales y asociadas al disturbio antrópico (área basal, altura, cobertura de copa, densidad de fustes, plántulas, árboles muertos y tocones) fueron comparadas entre grupos de manglar con un Análisis de Varianza Multivariado (MANOVA por sus siglas en inglés), posterior a la verificación de los supuestos de normalidad multivariada (pruebas de Mardia). Finalmente, la exploración espacial de las relaciones lineales entre dichas variables y los grupos identificados se realizó con un Análisis Discriminante Canónico (CDA por sus siglas en inglés). La contribución de cada variable se determinó mediante coeficientes estándares jerarquizados. Estos análisis se desarrollaron con rutinas en los paquetes Vegan 2.0 y Candisc 0.5 en la interfaz de R 2.14 (Friendly \& Fox, 2011; Oksanen, 2011; R Development Core Team, 2011).

\section{RESUlTADOS}

Durante este estudio se midieron un total de 2981 árboles correspondientes a siete especies; cuatro se consideran mangles verdaderos: $R$. mangle, L. racemosa, A. germinans y C. erectus; y tres como especies asociadas: P. aquatica, Pithecellobium dulce (Roxb.) Benth. y Coccoloba barbadensis Jacq. El $86.3 \%$ de los árboles presentaron un DAP $\leq 17.7 \mathrm{~cm}$, un área basal estimada de $36.5 \mathrm{~m}^{2} \mathrm{ha}^{-1}$, diámetro de copa promedio de $7.4 \mathrm{~m}^{2}$ y altura promedio de $15.5 \mathrm{~m}$.

\section{Clasificación y ordenación de las comunidades}

En el análisis de conglomerados se distinguieron cinco grupos de manglar basados en el Valor de Importancia de las especies entre los 40 sitios muestreados: Monoespecífico de $R$. mangle (Mono-Rm), Monoespecífico de A. germinans (Mono-Ag), Mixto de $P$. aquatica, Mixto de $L$. racemosa (Mix-Lr) y Mixto de C. erectus (Mix-Ce). El diagrama de ordenación por NMDS mostró correspondencia espacial con los grupos identificados por los conglomerados y con un bajo valor de estrés (0.08) (fig. 2). Esto reflejó una baja variabilidad entre las unidades de muestreo clasificadas como Mono-Rm, Mono-Ag y Mix-Pa, y con un relativo traslape en las comunidades Mix-Lr y Mix-Ce, señalando una mayor heterogeneidad en su composición. El ANOSIM indicó diferencias significativas en la estructura de los manglares por grupo $(\mathrm{R}=$ $0.96 ; \mathrm{p}=0.001)$ y por pares $(\mathrm{R}>0.71 ; \mathrm{p}<0.05)$.

Las variables ambientales con un mayor coeficiente de correlación con las dimensiones de ordenación NMDS fueron Salinidad (NMDS 2; R= -0.58; $<<0.0001$ ), Nivel de Inundación $(\mathrm{NMDS} 2 ; \mathrm{R}=0.64 ; \mathrm{p}<0.0001)$ y contenido de Materia Orgánica 30-60 cm (NMDS 1; $\mathrm{R}=$ $0.5 ; \mathrm{p}<0.01)$, en correspondencia con el gradiente espacial que separa a los grupos Mix-Pa y Mono-Ag (fig. 2). 


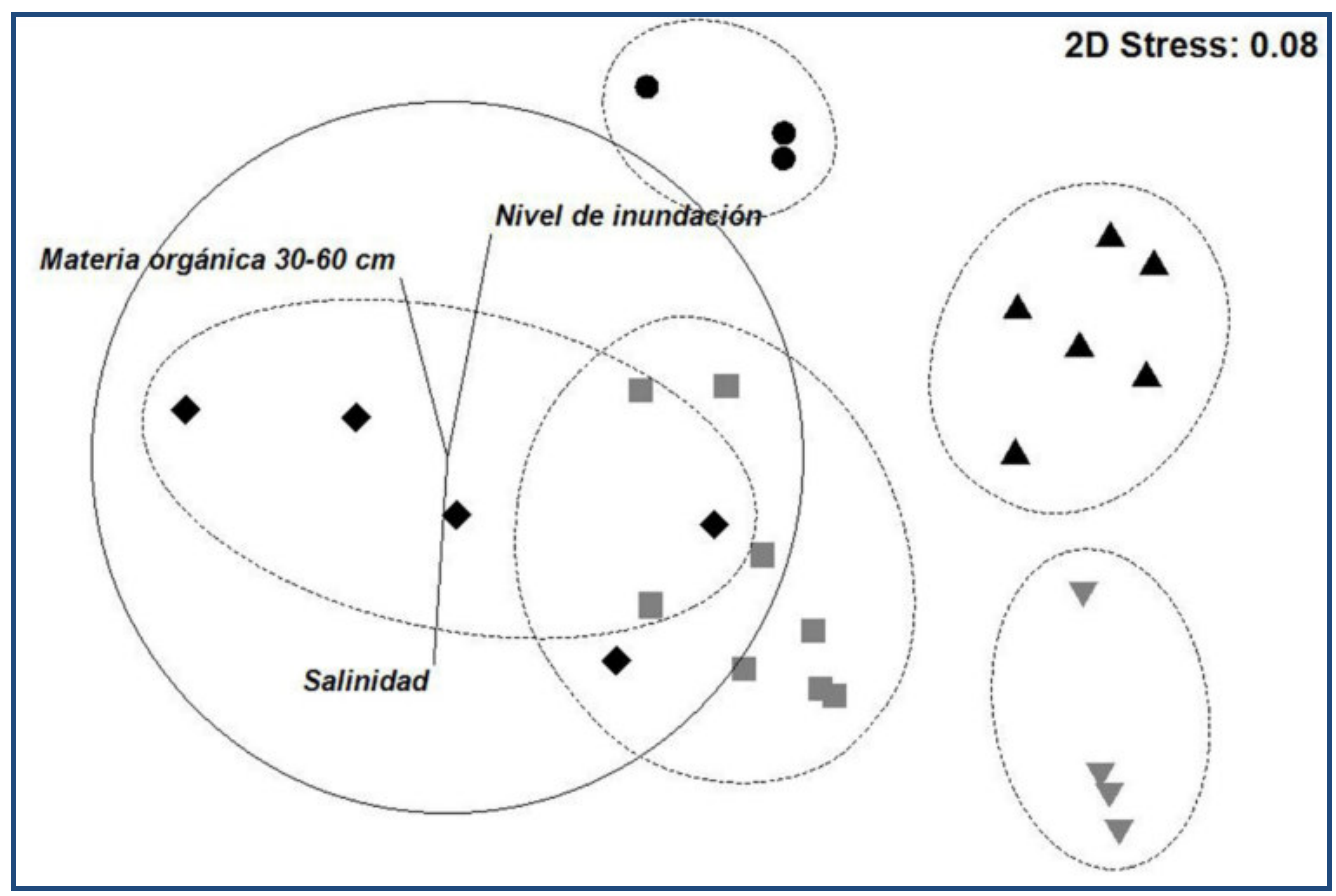

Fig. 2. Diagrama de ordenación por Escalamiento Multidimensional no Métrico para las unidades de muestreo. Las elipses punteadas representan los conglomerados identificados en la clasificación con similitud de $60 \%$. La longitud y dirección de los vectores representa la fuerza de la correlación de tres variables significativas (Pearson $>0.5)$ con respecto a un circulo unitario (línea continua). Grupos: $\boldsymbol{\Delta}($ Mono-Rm), $\nabla$ (Mono-Ag), $\bigcirc(\mathrm{Mix}-\mathrm{Pa}), \square(\mathrm{Mix}-\mathrm{Lr})$,

(Mix-Ce).

En el cuadro 1 se presentan los VIR y sus componentes para cada especie por grupo. El grupo Mono-Rm comprendió 13 sitios en donde $R$. mangle es dominante promedio. Fisiográficamente presenta correspondencia con formaciones tipo Borde y Ribereño. En esta comunidad se aprecia un subtipo de ocho sitios con $R$. mangle como especie única, y cuatro subtipos con cinco sitios en donde es dominante sobre L. racemosa, A. germinans y $P$. aquatica. El grupo Mono-Ag agrupó a nueve sitios con $A$. germinans como dominante promedio, correspondiendo a una formación tipo Cuenca. En un subtipo compuesto por siete sitios A. germinans es la especie única, mientras que en dos sitios domina sobre $R$. mangle y $L$. racemosa. El grupo Mix-Pa comprendió tres sitios con mayor dominancia promedio de la especie $P$. aquatica, de los cuales en dos sitios $R$. mangle se encuentra con dominancias mayores a $20 \%$ y en uno $P$. aquatica se presentó como especie única. Se trata de una formación fisiográfica tipo Ribereña. El grupo Mix-Lr incluyó 10 sitios con mayor dominancia promedio de L. racemosa, presentando un subgrupo de tres sitios monoespecíficos de esta especie, separándose un sitio donde domina sobre $R$. mangle y $A$. germinans. En otros dos subgrupos de esta comunidad, cuatro sitios presentaron rodales mixtos de L. racemosa/A. germinans y dos de L. racemosa/P. aquatica. El grupo presentó formaciones fisiográficas tipo Cuenca y Borde. La agrupación Mix-Ce agrupó a cinco sitios con dominancia mayor promedio de C. erectus. Incluyó subgrupos con tres sitios monoespecíficos de $C$. erectus, un sitio con dominancia de esta especie sobre L. racemosa y uno mixto de $C$. erectus/A. germinans (dominancia proporcional 38.5\%/37.8\%) con presencia de $L$. racemosa y $R$. mangle (dominancias de $16 \%$ y $7.7 \%$, respectivamente), siendo el sitio más heterogéneo del muestreo. Fisiográficamente son formaciones tipo Cuenca, aunque C. erectus se presentó también en comunidades terrestres periféricas al manglar. La distribución espacial de las UM clasificadas de acuerdo al tipo de comunidad se presenta en la figura 1. 
Cuadro 1. Valores de Importancia Relativos (VIR) de cada especie por grupo.

\begin{tabular}{|c|c|c|c|c|}
\hline $\begin{array}{c}\text { Tipo de comunidad/ } \\
\text { Especies }\end{array}$ & $\begin{array}{c}\text { Densidad } \\
(\%)\end{array}$ & $\begin{array}{c}\text { Dominancia } \\
(\%)\end{array}$ & $\begin{array}{c}\text { Frecuencia } \\
(\%)\end{array}$ & $\begin{array}{l}\text { VIR } \\
(\%)\end{array}$ \\
\hline \multicolumn{5}{|l|}{ Mono-Rm } \\
\hline Rhizophora mangle & 94.6 & 96.4 & 61.9 & 84.3 \\
\hline Laguncularia racemosa & 3.1 & 1.7 & 19 & 7.9 \\
\hline Avicennia germinans & 1.6 & 1.9 & 14.3 & 6 \\
\hline Pachira aquatica & 0.6 & 0.0 & 4.8 & 1.8 \\
\hline Total & 100 & 100 & 100 & 100 \\
\hline \multicolumn{5}{|l|}{ Mono-Ag } \\
\hline Avicennia germinans & 96.1 & 99.8 & 69.2 & 88.4 \\
\hline Rhizophora mangle & 3.7 & 0.2 & 23.1 & 9 \\
\hline Laguncularia racemosa & 0.2 & 0.0 & 7.7 & 2.6 \\
\hline Total & 100 & 100 & 100 & 100 \\
\hline \multicolumn{5}{|l|}{ Mix-Pa } \\
\hline Pachira aquatica & 89.8 & 78.2 & 33.3 & 67.1 \\
\hline Rhizophora mangle & 5.1 & 21.2 & 22.2 & 16.2 \\
\hline Laguncularia racemosa & 4.3 & 0.4 & 33.3 & 12.7 \\
\hline Pithecellobium dulce & 0.8 & 0.1 & 11.1 & 4 \\
\hline Total & 100 & 100 & 100 & 100 \\
\hline \multicolumn{5}{|l|}{ Mix-Lr } \\
\hline Laguncularia racemosa & 85.3 & 70.4 & 43.5 & 66.4 \\
\hline Avicennia germinans & 7.7 & 12.6 & 21.7 & 14 \\
\hline Rhizophora mangle & 4.3 & 11.9 & 8.7 & 8.3 \\
\hline Pachira aquatica & 2.4 & 5 & 21.7 & 9.7 \\
\hline Coccoloba barbadensis & 0.2 & 0.0 & 4.3 & 1.5 \\
\hline Total & 100 & 100 & 100 & 100 \\
\hline \multicolumn{5}{|l|}{ Mix-Ce } \\
\hline Conocarpus erectus & 85.6 & 79.5 & 50 & 71.7 \\
\hline Laguncularia racemosa & 13.3 & 12.2 & 30 & 18.5 \\
\hline Avicennia germinans & 0.7 & 6.9 & 10 & 5.9 \\
\hline Rhizophora mangle & 0.4 & 1.3 & 10 & 3.9 \\
\hline Total & 100 & 100 & 100 & 100 \\
\hline
\end{tabular}

\section{Estructura}

Las mayores densidades promedio $( \pm$ D.E.) de fustes se estimaron en los grupos Mix-Ce $(5,069 \pm$ $\left.3540 \mathrm{ha}^{-1}\right)$ y $\operatorname{Mix}-\operatorname{Lr}\left(2,967 \pm 1,148 \mathrm{ha}^{-1}\right)$, diferentes en comparación a los otros tipos de comunidades $(\mathrm{F}=7.2, \mathrm{p}<0.001$; fig. $3 \mathrm{~A})$. Los grupos Mono-Ag y Mix-Pa presentaron las mayores densidades de plántulas $\left(2,442 \pm 1,558\right.$ y $2,300 \pm 636 \mathrm{ha}^{-1}$, respectivamente) difiriendo significativamente $(\mathrm{F}=4.3, \mathrm{p}<0.001$; Fig. $3 \mathrm{~B})$ del resto de las comunidades. La mayor densidad de árboles muertos en pie se determinó en los grupos Mix-Ce $\left(208 \pm 192 \mathrm{ha}^{-1}\right)$ y Mix-Lr $(187 \pm$ $\left.122 \mathrm{ha}^{-1}\right)$, aunque estas diferencias no fueron significativas en relación a los demás grupos $(\mathrm{H}=$ 3.8, n.s; Fig. 3C). Para el caso de los árboles talados, se determinaron diferencias entre grupos ( $\mathrm{H}$ $=9.7, \mathrm{p}<0.05$; fig. 3D), con la mayor densidad de tocones estimada en Mix-Ce $\left(831 \pm 629\right.$ ha $\left.^{-1}\right)$. Los valores de área basal difirieron significativamente entre comunidades $(\mathrm{F}=6.8, \mathrm{p}<0.001$; fig. $3 \mathrm{E})$, mostrando la prueba post hoc una mayor afinidad entre los grupos Mono-Rm $\left(48.6 \pm 22.2 \mathrm{~m}^{2}\right.$ ha $\left.{ }^{-1}\right)$, Mono-Ag $\left(32.5 \pm 11.4 \mathrm{~m}^{2} \mathrm{ha}^{-1}\right)$ y Mix- $\operatorname{Lr}\left(41.4 \pm 30.9 \mathrm{~m}^{2} \mathrm{ha}^{-1}\right)$, siendo estos los valores más altos. En términos de altura máxima de los árboles, esta se encontró en $\operatorname{Mix}-\operatorname{Lr}(22 \pm 7.1 \mathrm{~m})$, en afinidad con las alturas estimadas en Mono-Rm, Mono-Ag y Mix-Pa, difiriendo significativamente $(\mathrm{F}=3.7, \mathrm{p}<0.05$; fig. $3 \mathrm{~F})$ de las Mix-Ce. La cobertura foliar presentó diferencias entre grupos $(\mathrm{F}=4.3, \mathrm{p}<0.01$; fig. $3 \mathrm{G})$, encontrándose la mayor en $\mathrm{Mix}-\mathrm{Pa}(16.6 \pm$ 
$12.3 \mathrm{~m}^{2}$ ), en correspondencia con la mayor cobertura relativa de dosel para esta misma comunidad $(100 \%)$, con diferencias respecto a los otros grupos $(\mathrm{F}=4.9, \mathrm{p}<0.01 ;$ fig. $3 \mathrm{H})$.

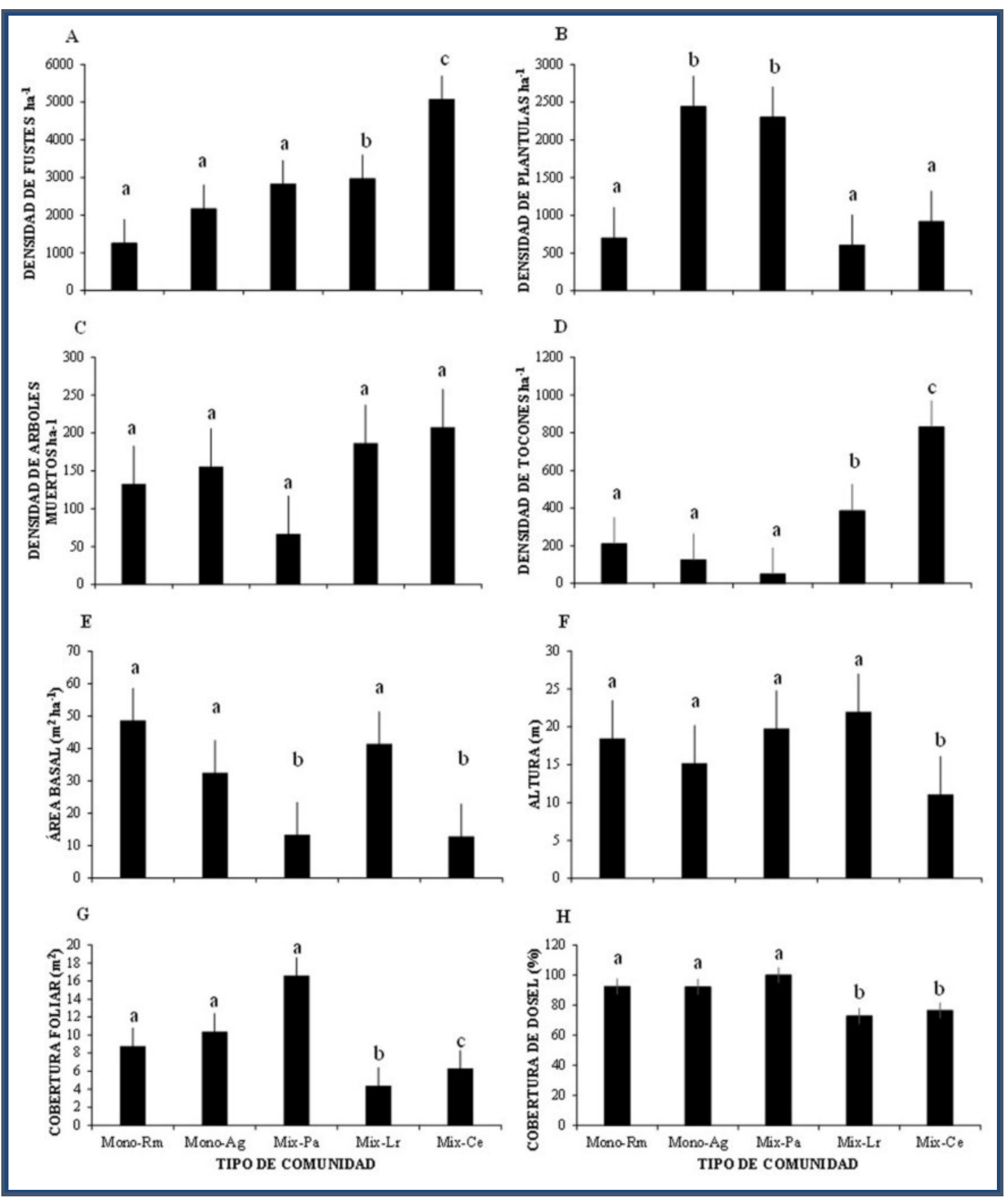

Fig. 3. Variación estructural de los grupos de manglar. Valores promedio \pm 1 E.E. de A) Densidad de fustes, B) Densidad de plántulas, C) Densidad de árboles muertos en pie, D) Densidad de tocones, E) Área basal, F) Altura, G) Cobertura foliar, H) Cobertura relativa del dosel. Para cada panel, letras diferentes indican diferencias significativas para cada variable entre tipos de comunidad $(p \leq 0.05)$. 


\section{Variables ambientales y su relación con la heterogeneidad estructural}

Con respecto a las condiciones físico-ambientales promedio para las dos estaciones climáticas, se observó que la salinidad fluctuó entre $26.7 \pm 16.8$ ups, en sitios con comunidades Mono-Ag, y $0.7 \pm 0.6$ ups en aquellos con comunidades Mix-Pa (fig. 4A). Así mismo, el mayor valor promedio de inundación $(55.2 \pm 32.4 \mathrm{~cm})$ se presentaron en este grupo, en contraste con el menor $(6.2 \pm 3.1 \mathrm{~cm})$ en sitios de Mix-Ce (fig. 4B). Los valores de $\mathrm{pH}$ fluctuaron entre $7 \pm 0.1$ (Mix-Pa) y $7.4 \pm 0.7$ (Mix-Ce; fig. 4C). La temperatura del aire al dentro de los sitios varió de $31.7 \pm 3.7^{\circ} \mathrm{C}$ en Mix-Ce a $29.1 \pm 2.5^{\circ} \mathrm{C}$ en Mix-Pa (fig. 4E). La mayor humedad relativa (75.9 \pm $5.5 \%)$ se registró dentro de los sitios de Mono-Rm, mientras que la menor $(69 \pm 0.9 \%)$ fue en Mix-Ce (fig. 4G). Los mayores porcentajes de materia orgánica en el suelo se encontraron en Mix-Pa (32.1 $\pm 9.6 \%$ de $0-30 \mathrm{~cm}$ y $23.6 \pm 9.5 \%$ de $30-60 \mathrm{~cm}$; fig. 4D); encontrándose los porcentajes más bajos en Mix-Ce $(7.7 \pm 0.7 \%$ de $0-30 \mathrm{~cm})$ y Mono-Ag $(4.6 \pm 4.4 \%$ de 30-60 cm; fig. 4H).

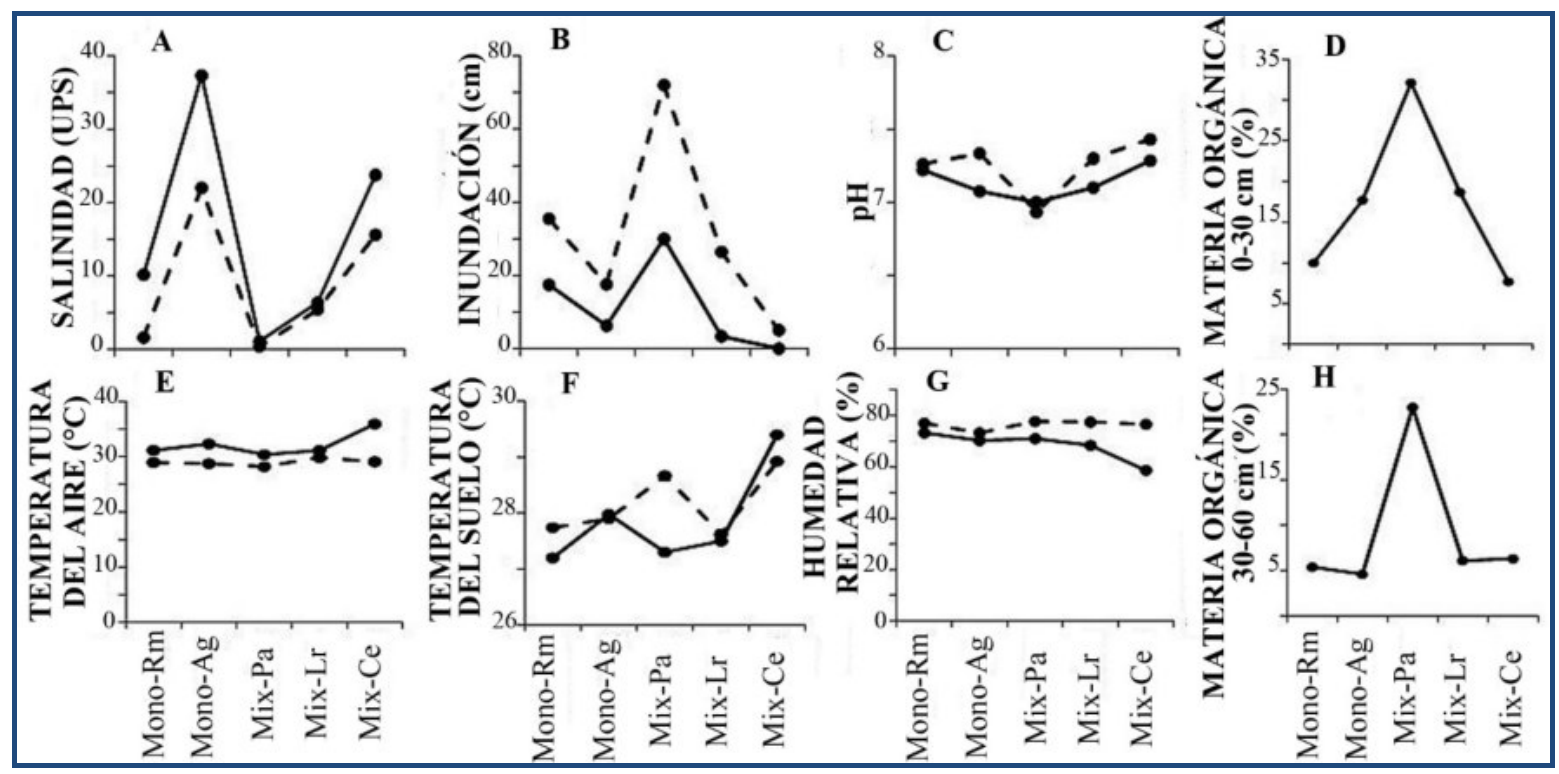

Fig. 4. Variables ambientales en las agrupaciones de manglar en la región sur del Soconusco, Chiapas, México. A) Salinidad, B) Nivel de inundación, C) pH, D) Porcentaje de materia orgánica $(0-30 \mathrm{~cm})$, E) Temperatura del aire, F) Temperatura del suelo, G) Humedad relativa, H) Porcentaje de materia orgánica $(30-60 \mathrm{~cm})$. Línea continua indica valores de la temporada seca. Línea punteada indica valores de la temporada lluviosa.

El MANOVA permitió corroborar significativamente la separación espacial entre las agrupaciones de manglar ( $\mathrm{T}$ de Pillai, $\mathrm{F}=3.04, \mathrm{p}<0.0001$ ), con lo que se justificó la exploración del patrón multivariado con el CDA. El CDA mostró que en dos funciones discriminantes se explica el $89 \%$ de la varianza total. Los coeficientes estandarizados $(>0.5)$ reflejaron una mayor importancia relativa de las variables Área basal, Altura y Diámetro de copa para la primera función; mientras que para la segunda función la variable de mayor contribución fue la de impacto antrópico Densidad de tocones (cuadro 2). En el diagrama de la ordenación se observó que los centroides para los grupos Mix-Pa y Mono-Ag se encontraron definidos por la Densidad de plántulas, mientras que el grupo Mix-Ce presentó relación espacial con las densidades de tocones y fustes. Para el caso del grupo Mono-Rm, se encontró mejor definida por la Altura. Es notable la relación inversa que presentan las variables Cobertura de 
dosel y Área basal con las variables asociadas a impacto antrópico Densidad de plántulas y Densidad de tocones, respectivamente (fig. 5).

Cuadro 2. Resumen de resultados del Análisis Discriminante Canónico y contribución de las variables estructurales a las funciones expresada en coeficientes estandarizados. *Variables que contribuyen más a la caracterización de los tipos de comunidad.

\begin{tabular}{|c|c|c|c|c|c|}
\hline \multirow{2}{*}{$\begin{array}{l}\text { Función } \\
\text { canónica }\end{array}$} & \multirow{2}{*}{$\begin{array}{c}\text { Valor } \\
\text { característico }\end{array}$} & \multicolumn{2}{|c|}{ Proporción } & \multirow{2}{*}{$\begin{array}{l}\text { Correlación } \\
\text { canónica }(\lambda)\end{array}$} & \multirow[t]{2}{*}{$\mathbf{p}>\mathbf{F}$} \\
\hline & & Valor & Acumulada & & \\
\hline Función 1 & 2.204 & 54.54 & 54.54 & 0.089 & 0.001 \\
\hline Función 2 & 1.394 & 34.50 & 89.05 & 0.286 & 0.001 \\
\hline \multirow{2}{*}{\multicolumn{2}{|c|}{ Variables estructurales }} & \multicolumn{2}{|c|}{ Coeficientes estandarizados } & & \\
\hline & & Función 1 & Función 2 & & \\
\hline Densidad de f & & 0.305 & 0.268 & & \\
\hline Área basal & & $0.878^{*}$ & 0.359 & & \\
\hline Densidad de $t$ & & 0.209 & $0.925^{*}$ & & \\
\hline Densidad de á & nuertos & -0.411 & -0.418 & & \\
\hline Densidad de $p$ & & 0.402 & -0.209 & & \\
\hline Altura & & $-0.945 *$ & 0.369 & & \\
\hline Cobertura de & & $-0.761 *$ & 0.277 & & \\
\hline
\end{tabular}

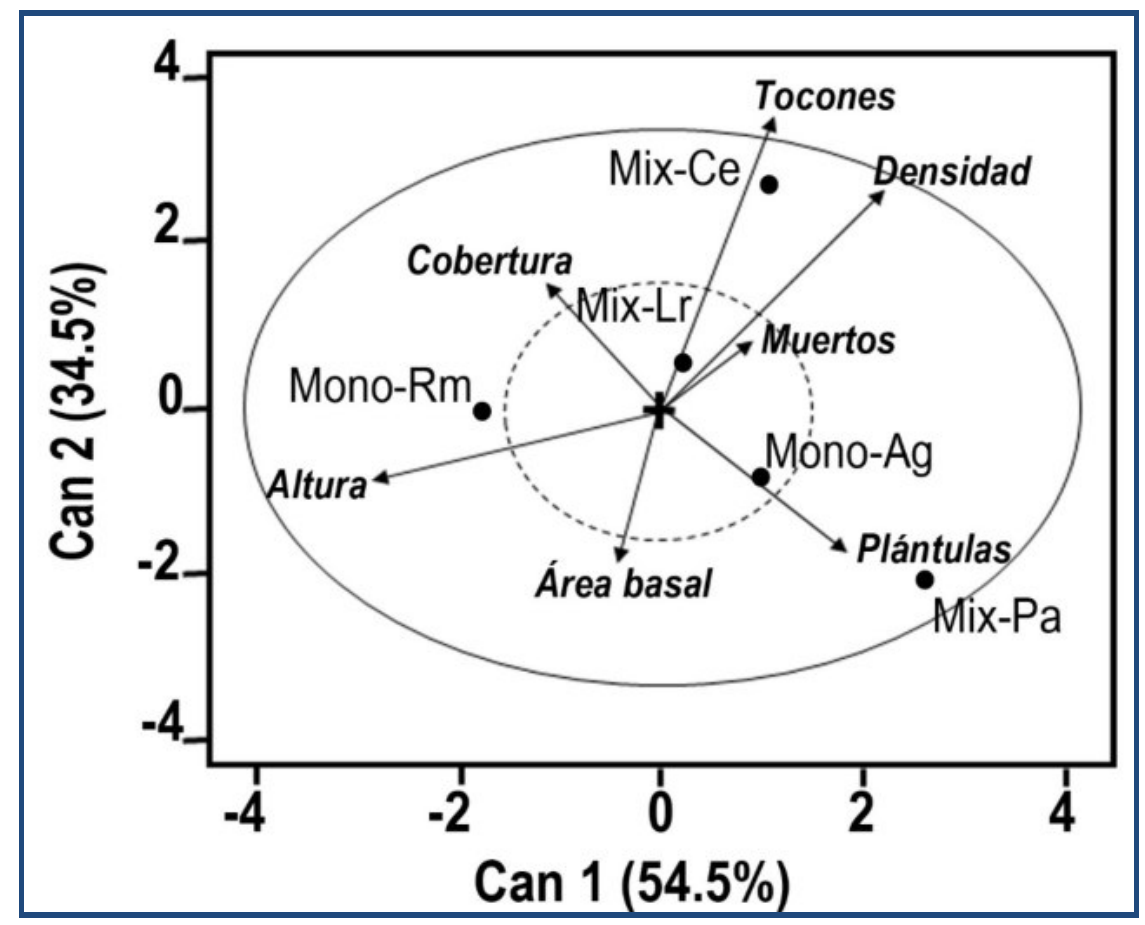

Fig. 5. Diagrama del Análisis Discriminante Canónico de las variables estructurales de los manglares. Los puntos en color negro representan los centroides calculados para grupo identificado. Los coeficientes de correlación para cada variable se muestran como vectores. 


\section{DISCUSIÓN}

Las clasificaciones en comunidades de manglar se han basado a partir de dos enfoques principales: fisiográfico-estructural y geomorfológico (Lugo \& Snedaker, 1974; Thom, 1984). Como se demuestra en este estudio, los rasgos diferenciales de dominancia y composición permiten delinear agrupaciones locales de manglares que favorecen su manejo regional y la valoración de sus servicios ecosistémicos (Duke, 2006; Ewel et al., 1998; González-Zamorano et al., 2013; Trejo-Torres et al., 1993; Urrego, Polanía, Buitrago, Cuartas, \& Lema, 2009).

En esta región se encontraron las cuatro especies de mangles más comunes para México (Pennington \& Sarukhán, 2005), presentes en distinto grado de dominancia en los cinco tipos grupos identificados. Las marcadas diferencias en su estructura, pueden indicar un traslape de requerimientos ambientales y tolerancia a cierto grado de estrés ambiental (Upkong, 1995). La interrelación de variables como la inundación, salinidad, fertilidad y la saturación del suelo define gradientes ambientales en las áreas intermareales que dirige una zonación o estratificación en la distribución de los manglares, patrón que cuenta con una sólida evidencia experimental (Bunt, 1996; Krauss et al., 2008). Desde un enfoque ecofisiológico, los mangles pueden superponerse en su rango de tolerancia a la interacción de factores ambientales o al estrés por impacto antrópico, favoreciendo la distribución de especies en un nicho funcional estrecho (Ball, 1988; Barnuevo \& Asaeda, 2018). Estos paradigmas sustentan el resultado obtenido en nuestro estudio, que demuestra la conformación de comunidades de manglar diferenciables en composición, dominancia y estructura en respuesta a las condiciones ambientales.

Se ha demostrado que las especies típicas de mangles presentes exhiben diferentes requerimientos ambientales en respuesta a su historia evolutiva, coexistiendo mediante un uso diferenciado de los recursos disponibles en el suelo bajo marcados gradientes de salinidad (Krauss et al., 2008). Por ejemplo, mientras que $R$. mangle puede desarrollarse con éxito ecológico en salinidades cercanas a 35\%, A. germinans puede encontrar un óptimo desarrollo en ambientes hipersalinos; por otro lado, aunque L. racemosa prefiere sitios menos salinos, su gran plasticidad fenotípica le permite colonizar una mayor diversidad de ambientes incluyendo los sujetos a perturbación. Para el caso de $C$. erectus, la especie tiende a distribuirse en hábitats con menor influencia mareal, periféricos al manglar y en contacto con la vegetación terrestre (Agudelo et al., 2015; Montes-Cartas et al., 1999; Urrego et al., 2009).

En este estudio, las especies $A$. germinans y $R$. mangle presentaron mayor tendencia a formar rodales monoespecíficos, probablemente como una adaptación intrínseca a condiciones ambientales específicas (López, Barreto, \& Conde, 2011). Mientras $R$. mangle tolera mejor los suelos con mayor grado de anoxia, A. germinans prospera en depresiones propensas a la hipersalinidad (Arreola-Lizárraga, Flores-Verdugo, \& Ortega-Rubio, 2004). En este estudio se observó que en sitios con una alta dominancia de $R$. mangle, prevalecen condiciones de menor salinidad, lo que puede reflejar una mayor frecuencia de inundación y menor evaporación (Agudelo et al., 2015; Strauch, Cohen, \& Ellmore, 2012). Hacia el interior de la planicie costera, la variación microtopográfica, permite el desarrollo de A. germinans en cuencas de inundación somera, con una amplitud de marea reducida e incremento de salinidad por mayor tasa de evaporación. L. racemosa presentó una mayor plasticidad de desarrollo en rangos variantes de salinidad e inundación, y una mayor tendencia a formar rodales mixtos con elevado desarrollo estructural.

En este estudio se observó que el grupo Mono-Rm presenta atributos estructurales similares a los señalados por Tovilla-Hernández \& Romero-Berny (2012) en comunidades afines descritas en la zona de Panzacola-El Hueyate al centro del Soconusco (área basal: $45 \mathrm{~m}^{2} \mathrm{ha}^{-1}$; altura: 22 m; densidad: 2352 fustes ha $^{-1}$ ). En contraste, los mismos autores describen rodales arbustivos de $R$. mangle en el istmo sur de Tehuantepec, Oaxaca, localizados a poco más de $250 \mathrm{~km}$ al norte del Soconusco, y que presentan bajo desarrollo estructural (área basal: $4.32 \mathrm{~m}^{2} \mathrm{ha}^{-1}$; altura: 
$4.18 \mathrm{~m}$; densidad: 2701 fustes $\mathrm{ha}^{-1}$ ). Para el caso de los grupos monoespecíficos de $A$. germinans, en este estudio se presentan valores estructurales más altos, aunque en menores densidades. Por ejemplo, a lo largo del Pacífico mexicano se ha registrado rangos de área basal de 11.9-16.9 $\mathrm{m}^{2} \mathrm{ha}^{-1}$ y alturas medias de 4.5-7 m en ambientes semiáridos; y áreas basales de 12.5-29.6 $\mathrm{m}^{2} \mathrm{ha}^{-1}$ y alturas de 7.5-8.8 m en localidades sub-húmedas (Flores-Verdugo, González-Farías, Segura-Zamorano, \& Ramírez-García, 1992). Estos patrones pueden reflejar árboles de mayor tamaño en menor densidad y altas densidades de plántulas, indicando rodales maduros, y en respuesta a sitios menos inundados en donde la salinidad puede ser mayor (Cintrón \& Schaeffer, 1984; Rodríguez-Ramírez, Nivia-Ruíz, \& Garzón-Ferreira, 2004). Los sitios de Mix-Lr presentaron altos valores en las variables de densidad, área basal y altura, similares en composición y estructura a los manglares de la zona de Manialtepec, Oaxaca (área basal: $49.1 \mathrm{~m}^{2} \mathrm{ha}^{-1}$; altura: $17.3 \mathrm{~m}$; densidad: 2,367 fustes ha ${ }^{-1}$ ) (Tovilla-Hernández \& RomeroBerny, 2012). Debido a los altos requerimientos de luz de la especie, las mayores densidades en L. racemosa, han sido asociadas a procesos de sucesión posteriores a disturbios como la apertura de claros (Jiménez \& Soto, 1985; McGowan et al., 2010; Montes-Cartas et al., 1999). Para los grupos Mix-Ce, se encontraron elevadas densidades, en concordancia con los bosques descritos en el estado de Guerrero (Tovilla-Hernández \& de la Lanza-Espino, 1999), quienes registran plantas jóvenes de esta especie con DAP de entre 5 y $20 \mathrm{~cm}$.

El manglar con alta dominancia de $P$. aquatica se presenta en áreas restringidas del Soconusco, sin que se haya encontrado un tipo de comunidad similar al norte de esta región en la costa del Pacífico. Respecto a su estructura, Montes-Cartas et al. (1999) encontraron comunidades de $R$. mangle- $P$. aquatica con elevado desarrollo en la Reserva de la Biósfera La Encrucijada, aproximadamente a $20 \mathrm{~km}$ al noroeste de los sitios de este estudio, con alturas promedio de entre 20 y $30 \mathrm{~m}$, rasgo probablemente asociado a un menor estrés por salinidad. En el Golfo de México $P$. aquatica se ha registrado como un componente más común de la vegetación de manglar. En Laguna Chica, Veracruz, se describen asociaciones de $P$. aquatica y manglar con área basal de $47.44 \mathrm{~m}^{2} \mathrm{ha}^{-1}$, altura promedio de $12 \mathrm{~m}$ y densidad de 2400 fustes ha $^{-1}$ (InfanteMata, Moreno-Casasola, Madero-Vega, Castillo-Campos, \& Warner, 2011). En el Soconusco la distribución de esta especie parece asociarse a ambientes de baja salinidad (0-1.7 ups). En el Golfo de México se han registrado comunidades de $P$. aquatica-R. mangle en condiciones de hasta 18.7 ups de salinidad superficial, aunque esto no necesariamente puede indicar estrés a nivel de raíces (Infante-Mata, Moreno-Casasola, \& Madero-Vega, 2014). Es evidente que $P$. aquatica comparte diversas adaptaciones biológicas y ecológicas con los manglares, mismas que favorecen su desarrollo en sitios con inundación permanente, los mecanismos que le permiten tolerar la salinidad aún son poco conocidos.

Como se ha analizado en otras áreas de manglar, se observa un gradiente abiótico, siendo determinantes la salinidad y el nivel de inundación, análisis que respalda la hipótesis de una respuesta estructural en manglares a variaciones de microtopografía e hidroperiodo (FloresVerdugo et al., 2007; Méndez-Linares et al., 2007), o a cambios en el régimen anual de precipitación por periodos prolongados (Agraz-Hernández et al., 2015). Otra variable correlacionada puede ser el carbono, almacenado en forma de materia orgánica en suelos de manglar. El mayor porcentaje se obtuvo en muestras de suelo de 0-30 cm en grupos de Mix-Pa, aunque el carbono puede ser más significativo a nivel de rizoma a profundidad media $(>30$ $\mathrm{cm})$. En relación con otras comunidades forestales asociadas a humedales, esto puede deberse a una elevada producción de hojarasca y un mayor tiempo de inundación durante todo el año, lo cual limita la descomposición de materia orgánica por una baja difusión de oxígeno (Whiting \& Chanton, 2001).

El pH en áreas de manglar normalmente se mantiene en valores ligeramente básicos, situación observada en este estudio. Es particularmente de interés el seguimiento de la humedad relativa y la temperatura del aire. Tovilla-Hernández et al. (2001) registraron para un periodo anual, un aumento de $11^{\circ} \mathrm{C}$ en la temperatura y menor humedad dentro de un manglar a causa de la deforestación, demostrando que las condiciones ambientales locales pueden ser modificadas por 
un agente de perturbación. No obstante, es necesario tener en cuenta que estas variables pueden presentar marcadas fluctuaciones a lo largo del día, por lo que se recomienda estandarizar su medición.

Con respecto a algunos patrones estructurales, se ha demostrado que la altura y el área basal muestran una relación inversa con la salinidad. Estos atributos disminuyen progresivamente con el incremento de la salinidad y un decrecimiento en la disponibilidad de nutrientes (López et al., 2011; Lugo \& Snedaker, 1974). El bajo aporte de agua dulce implica una menor cantidad de nutrientes y la acumulación de sales en el sedimento, aspectos reflejados en la estructura de las comunidades. Montes-Cartas et al. (1999) encontraron variación en la composición y altura de los manglares en un gradiente latitudinal: los manglares de la costa norte de Chiapas con menor aporte de escorrentías presentan menor desarrollo estructural que los de la costa sur, en donde prevalece una mayor precipitación y número de ríos que drenan hacia la costa.

Una variable de perturbación en este estudio que presenta relación significativa con la comunidad Mix-Ce es la densidad de tocones. Tovilla-Hernández \& De la Lanza-Espino (1999) registran que es una especie sobreexplotada en México. Los mismos autores señalan que las especies con mayor preferencia de uso son $C$. erectus, $L$. racemosa y en menor grado $R$. mangle. Su amplio uso como leña o material de construcción conduce a una tala selectiva. Por ejemplo, en el sistema lagunar de Alvarado, Veracruz, el elevado número de mangles extraídos ha conducido a alteraciones en la dominancia del bosque (Rodríguez-Zúñiga et al., 2011). Sin embargo, se reconoce que los efectos a largo plazo de la remoción de individuos en la estructura del manglar aún son poco conocidos. A pesar de la implementación de diversos instrumentos legales para reducir el consumo de madera de mangle (Gobierno de Chiapas, 2010), su uso es extenso y poco regulado.

La estimación de la densidad de plántulas permitió una aproximación al proceso de regeneración natural. Este proceso se relaciona con la cantidad de luz que penetra al sotobosque, y, según la especie, puede afectar la sobrevivencia de las plántulas (Duke, 2001). La luz puede estar relacionada con la cobertura relativa del dosel, la caída de árboles muertos y la extracción selectiva. En este trabajo se encontró relación entre la cobertura del dosel y la densidad de plántulas. Estas dos comunidades presentan las mayores densidades de plántulas registradas en este estudio. La mayor densidad de plántulas se encontró en grupos de Mix-Ag, la cual presentó un dosel cerrado (92.2\%). Pinto-Nolla (1999) indica que A. germinans presenta un mayor crecimiento de plántulas establecidas durante el primer año, y en cierto grado intolerante a grandes cantidades de luz, presentando un mejor desarrollo bajo el dosel cerrado. Los grupos Mix-Lr y Mix-Ce presentaron los doseles menos cerrados para el área de estudio (< $76.5 \%$ ). Así mismo presentan menor densidad de plántulas, asumiéndose en parte como una consecuencia del impacto por extracción. Se ha mencionado que L. racemosa presenta tasas más altas de germinación y sobrevivencia bajo condiciones de luz, lo que puede reflejar un mayor éxito en competencia interespecífica (Blanco, Estrada, Ortiz, \& Urrego, 2012; PintoNolla, 1999).

El grupo Mix-Pa, desarrolla un dosel completamente cerrado y alta densidad de plántulas. Se ha demostrado que $P$. aquatica es una especie de alta plasticidad fenotípica, adaptable a condiciones de alta y baja intensidad lumínica; pudiendo las plántulas presentar menor desarrollo, pero alta sobrevivencia bajo condiciones limitadas de luz (Infante-Mata \& MorenoCasasola, 2005).

En la mayoría de los grupos de manglar identificados en este estudio, se encontraron bajas densidades de árboles muertos en pie. Jiménez y Soto (1985) señalan que la tasa de mortalidad natural en mangles maduros, al igual que en la mayoría de los árboles tropicales, es muy baja. Los factores naturales que influyen en la mortalidad de los manglares, son la competencia intraespecífica, herbivoría, enfermedades endémicas y senescencia. Es un proceso denso dependiente y que afecta a propágulos, plántulas y árboles con distinto grado de madurez, 
aunque se ha observado que la mayor mortalidad ocurre en plántulas y arbolado joven. Otro tipo de mortalidad natural es causada por fenómenos meteorológicos, tales como tormentas y huracanes que pueden provocar eventos de mortalidad masiva, afectando principalmente a comunidades tipo cuenca (Smith et al., 2009).

De manera general, se considera que la región del Soconusco está sujeta a diversos impactos, como la contaminación por residuos sólidos, aguas residuales y agroquímicos, además de la deposición y sedimentación de cuerpos de agua como consecuencia de la erosión y transporte de sedimentos de áreas deforestadas en la cuenca media y alta. A pesar de la presencia de áreas naturales protegidas, la falta de un plan de gestión y regulación del uso de recursos, tiene una influencia determinante en la degradación de la vegetación. Se considera importante considerar las características estructurales de cada tipo de manglar, a fin de diseñar una zonificación apropiada de las áreas protegidas acorde con su dinámica espacial, y proponer medidas de rehabilitación y restauración de ecosistemas y otras medidas de mitigación de impactos. Es conveniente establecer un control permanente de los bosques de manglares y promover el desarrollo de políticas que fortalezcan la gestión integrada de la cuenca costera del estado de Chiapas.

\section{AGRADECIMIENTOS}

A CONABIO por el financiamiento asignado al proyecto "Inventario y monitoreo del estado actual de los bosques de manglar de Chiapas y Oaxaca" (FN005) y a CONACYT por la beca otorgada al primer autor (Núm. 239310). Este trabajo se deriva parcialmente de la tesis de maestría de E.I. Romero-Berny "Manglares del Soconusco: estructura y cambios a nivel de paisaje", en el programa de posgrado en Recursos naturales y Desarrollo rural de El Colegio de la Frontera Sur. Agradecemos especialmente el apoyo en campo brindado por Felipe Ovalle, Juan C. De la Presa, Gerardo de la Cruz, Alejandro Lang y Norberto Gómez, así como a los revisores anónimos por los comentarios y sugerencias hechas a este artículo.

\section{LiTERATURA CITADA}

Agraz-Hernández, C. M., Chan-Keb, C. A., Iriarte-Vivar, S., Posada-Venegas, G., VegaSerratos, B., \& Osti-Sáenz, J. (2015). Phenological variation of Rhizophora mangle and ground water chemistry associated to changes of the precipitation. Hidrobiológica, 25(1), 49-61. Retrieved from http://www.scielo.org.mx/scielo.php?script $=$ sci_arttext\&pid=S0188-88972015000100006\&lng=es\&nrm=iso\&tlng=en

Agraz-Hernández, C. M., \& Flores-Verdugo, F. (2014). Diagnóstico del impacto y lineamientos básicos para los programas de mitigación y manejo de humedales. In A. V. Botello, J. Rendón von Osten, J. Benítez, \& G. Gold-Bouchot (Eds.), Golfo de México. Contaminación e impacto ambiental: diagnóstico y tendencias (3rd ed., pp. 597-606). Campeche: CINVESTAV-IPN, UAC-IEPOMEX, UNAM-ICMYL.

Agudelo, C. M., Bolívar, J., Polanía, J., Urrego, L. E., Yepes, A., \& Sierra, A. (2015). Estructura y composición florística de los manglares de la bahía de Cispatá, Caribe colombiano. Revista de Biología Tropical, 63(4), 1137-1147. Retrieved from https://revistas.ucr.ac.cr/index.php/rbt/article/view/17076/21738

Anderson, M. J., Gorley, R. N., \& Clarke, K. N. (2008). PERMANOVA+ for PRIMER: Guide to software and statistical methods (1st ed.). Plymouth: PRIMER-E Ltd.

Arreola-Lizárraga, J. A., Flores-Verdugo, F., \& Ortega-Rubio, A. (2004). Structure and litterfall of an arid mangrove stand on the Gulf of California, Mexico. Aquatic Botany, 79(2), 137-143. https://doi.org/https://doi.org/10.1016/j.aquabot.2004.01.012

Ball, M. C. (1988). Ecophysiology of mangroves. Trees, 2(3), 129-142. https://doi.org/10.1007/BF00196018 
Barnuevo, A., \& Asaeda, T. (2018). Integrating the ecophysiology and biochemical stress indicators into the paradigm of mangrove ecology and a rehabilitation blueprint. PLOS ONE, 13(8), e0202227. https://doi.org/10.1371/journal.pone.0202227

Blanco, J. F., Estrada, E. A., Ortiz, L. F., \& Urrego, L. E. (2012). Ecosystem-Wide Impacts of Deforestation in Mangroves: The Urabá Gulf (Colombian Caribbean) Case Study. ISRN Ecology, 2012, 1-14. https://doi.org/10.5402/2012/958709

Breedlove, D. (1981). Introduction to the flora of Chiapas (1st ed.). San Francisco: California Academy of Sciences.

Bunt, J. S. (1996). Mangrove zonation: An examination of data from seventeen riverine estuaries in tropical Australia. Annals of Botany, 78(3), 333-341. https://doi.org/10.1006/anbo.1996.0128

Cintrón, G., \& Schaeffer, Y. (1984). Methods for studying mangrove structure. In S. C. Snedaker \& J. G. Snedaker (Eds.), The mangrove ecosystem: research method (1st ed., pp. 91-113). Bungay: UNESCO.

de la Lanza-Espino, G., Ortiz-Pérez, M. A., \& Carbajal-Pérez, J. L. (2013). Diferenciación hidrogeomorfológica de los ambientes costeros del Pacífico, del Golfo de México y del Mar Caribe. Investigaciones Geográficas, 81, 33-50. https://doi.org/dx.doi.org/ 10.14350/rig.33375

Domínguez-Cadena, R., Riosmena-Rodríguez, R., \& León-de la Luz, J. L. (2016). Forest Structure and Species Composition of Mangroves in the Eastern Baja California Peninsula: The Role of Microtopography. Wetlands, 36(3), 515. https://doi.org/10.1007/s13157-016-0760-9

Duke, N. C. (2001). Gap creation and regenerative processes driving diversity and structure of mangrove ecosystems. Wetland Ecology and Management, 9(3), 257-269. https://doi.org/10.1023/A:1011121109886

Duke, N. C. (2006). Australia's mangroves. The authoritative guide to Australian's mangrove plants (1st ed.). Queensland: University of Queensland.

Ewel, K. C., Twilley, R. R., \& Ong, J. E. (1998). Different kind of mangrove forests provided different goods and services. Global Ecology and Biogeography, 7(1), 83-94. https://doi.org/10.2307/2997700

Fernández, B. E. (2004). La producción agropecuaria en el Soconusco e intercambio con Centroamérica. In J. Sánchez \& R. Járquin (Eds.), La frontera sur: reflexiones sobre el Soconusco, Chiapas y sus problemas ambientales, sociales y productivos (1st ed., pp. 185-200). México D.F.: ECOSUR.

Flores-Verdugo, F., de la Lanza-Espino, G., Contreras-Espinosa, F., \& Agraz-Hernández, C. M. (2001). The Tropical Pacific Coast of Mexico. In U. Seeliger \& B. Kjerfve (Eds.), Coastal Marine Ecosystems of Latin America (1st ed., pp. 307-314). Heidelberg: Springer-Verlag. https://doi.org/10.1007/978-3-662-04482-7_21

Flores-Verdugo, F., González-Farías, F., Segura-Zamorano, D., \& Ramírez-García, P. (1992). Mangrove ecosystem of the Pacific coast of Mexico: distribution, structure, litterfall and detritus dynamics. In E. Seeliger (Ed.), Coastal Plant Communities of Latin America (1st ed., pp. 269-288). New York: Academic Press Inc. https://doi.org/10.1016/C2009-0-02633-6

Flores-Verdugo, F., Moreno-Casasola, P., Agraz-Hernández, C. M., López-Rosas, H., BenítezPardo, D., \& Travieso-Bello, A. C. (2007). La topografía y el hidroperíodo: dos factores que condicionan la restauración de los humedales costeros. Boletín de La Sociedad Botánica de México, 80((Suplemento)), 33-47. https://doi.org/ 10.17129/botsci.1755

Friendly, M., \& Fox, J. (2011). Candisc: Generalized Canonical Discriminant Analysis. Retrieved January 6, 2012, from www.cran.r-project.org/package $=$ candisc

Gobierno de Chiapas. (2010). Programa de Manejo de la Zona Sujeta a Conservación Ecológica El Cabildo-Amatal. (1st ed.). Tuxtla Gutiérrez: SEMAVI.

González-Zamorano, P., Lluch-Cota, S. E., \& Nava-Sánchez, E. H. (2013). Relation between the Structure of Mangrove Forests and Geomorphic Types of Lagoons of the Baja California Peninsula. Journal of Coastal Research, 29(1), 173-181. 
https://doi.org/10.2307/23353580

Hogarth, P. J. (2007). The Biology of Mangroves and Seagrasses (3rd ed.). Oxford: Oxford University Press. https://doi.org/10.1093/acprof:oso/9780198716549.001.0001

Infante-Mata, D., \& Moreno-Casasola, P. (2005). Effect of in situ storage, light, and moisture on the germination of two wetland tropical trees. Aquatic Botany, 83(3), 206-218. https://doi.org/10.1016/j.aquabot.2005.06.009

Infante-Mata, D., Moreno-Casasola, P., \& Madero-Vega, C. (2014). ¿Pachira aquatica, un indicador del límite del manglar? Revista Mexicana de Biodiversidad, 85, 143-160. https://doi.org/10.7550/rmb.32656

Infante-Mata, D., Moreno-Casasola, P., Madero-Vega, C., Castillo-Campos, G., \& Warner, B. G. (2011). Floristic composition and soil characteristics of tropical freshwater forested wetlands of Veracruz on the coastal plain of the Gulf of Mexic. Forest Ecology and Management, 262(8), 1514-1531. https://doi.org/10.1016/ j.foreco.2011.06.053

Jiménez, J., \& Soto, R. (1985). Patrones regionales en la estructura y composición florística de los manglares de la Costa Pacífica de Costa Rica. Revista de Biología Tropical, 33(1), 25-37. https://doi.org/10.1134/1.1595201

Krauss, K. W., Lovelock, C. E., McKee, K. L., López-Hoffman, L., Ewe, S. M. L., \& Sousa, W. P. (2008). Environmental drivers in mangrove establishment and early development: A review. Aquatic Botany, 89(2), 105-127. https://doi.org/10.1016/j.aquabot.2007.12.014

López-Portillo, J., \& Ezcurra, E. (1989). Response of Three Mangroves to Salinity in Two Geoforms. Functional Ecology, 3(3), 355-361. https://doi.org/10.2307/2389376

López, B., Barreto, M. B., \& Conde, J. E. (2011). Caracterización de los manglares de zonas semiáridas en el noroccidente de Venezuela. Interciencia, 36(12), 888-893. Retrieved from http://www.redalyc.org/articulo.oa?id=33921507004

Lugo, A. E., \& Snedaker, S. C. (1974). The Ecology of Mangroves. Annual Review of Ecology and Systematics, 5, 39-64. https://doi.org/10.1146/annurev.es.05.110174.000351

Madrigal, A. (1994). Ordenacion de Montes Arbolados. Madrid: ICONA.

McGowan, T., Cunningham, S. L., Guzmán, H. L., Mair, J. M., Guevara, J. M., \& Betts, T. (2010). Mangrove forest composition and structure in Las Perlas Archipelago, Pacific Panama. Revista de Biología Tropical, 58(3), 857-869. Retrieved from http://www.redalyc.org/articulo.oa?id=44918839006

Méndez-Linares, A. P., López-Portillo, J., Hernández-Santana, J. R., Ortiz-Pérez, M. A., \& Oropeza-Orozco, O. (2007). The mangrove communities in the Arroyo Seco deltaic fan, Jalisco, Mexico, and their relation with the geomorphic and physical-geographic zonation. Catena, 70(2), 127-142. https://doi.org/10.1016/j.catena.2006.05.010

Montes-Cartas, C. G., Castillo-Argüero, S., \& López-Portillo, J. (1999). Distribución del manglar en cuatro sistemas lagunares en la costa de Chiapas, México. Boletín de La Sociedad Botánica de México, 64, 25-34. https://doi.org/10.17129/botsci.1579

Oksanen, J. (2011). Vegan: an introduction to ordination. Retrieved January 6, 2012, from $\mathrm{http}: / /$ www.cran.r-project.org/package= vegan

Ovalle-Estrada, F., \& Vásquez-Lule, A. D. (2009). Caracterización del sitio de manglar Lagunas Cabildo-Amatal-Gancho Murillo. In CONABIO (Ed.), Sitios de manglar con relevancia biológica y con necesidades de rehabilitación ecológica (1st ed., pp. 1-18). México D.F.: CONABIO. Retrieved from http://www.conabio.gob.mx/ conocimiento/manglares/doctos/caracterizacion/PS27_Lagunas_Cabildo_Amatal_Gan cho_Murillo_caracterizacion.pdf

Pennington, T. D., \& Sarukhán, J. (2005). Árboles tropicales de México. Manual para la identificación de las principales especies (3rd ed.). México D.F.: UNAM, FCE.

Pinto-Nolla, F. (1999). Sucesión y fisonomía de los manglares de Colombia. Revista de La Academia Colombiana de Ciencias Exactas, Físicas y Naturales, 23, 141-161.

Pool, D. J., Snedaker, S. C., \& Lugo, A. E. (1977). Structure of Mangrove Forests in Florida, Puerto Rico, Mexico, and Costa Rica. Biotropica, 9(3), 195-212. https://doi.org/10.2307/2387881. Retrieved from http://www.cran.r-project.org

R Development Core Team. (2011). R: a language and environment for statistical computing. 
Ramírez-García, P., \& Segura-Zamorano, D. (1994). Ordenación de la vegetación de manglar de la laguna Panzacola, Chiapas. Serie Grandes Temas de La Hidrobiología:Los Sistemas Litorales, 2, 105-113. Retrieved from https://docplayer.es/70649375Ordenacion-de-la-vegetacion-de-manglar-de-la-laguna-panzacola-chiapas.html

Ramos-Durón, F. J., Quiróz-Flores, A. J., Ramírez García-Armora, J. P., \& Lot-Helgueras, A. (2004). Manual de hidrobotánica. Muestreo y análisis de la vegetación acuática (1st ed.). México D.F.: AGT Editor.

Rodríguez-Ramírez, A., Nivia-Ruíz, J., \& Garzón-Ferreira, J. (2004). Características estructurales y funcionales del manglar de Avicennia germinans en la Bahía de Chengue (Caribe Colombiano). Boletín de Investigaciones Marinas y Costeras, 33, 223-244. Retrieved from http://www.scielo.org.co/pdf/mar/v33n1/v33n1a12.pdf

Rodríguez-Zúñiga, M. T., Ramírez-García, P., \& Gutiérrez-Granados, G. (2011). Efectos de la extracción no controlada de madera sobre la comunidad y estructura de tamaños de los manglares de Alvarado, Veracruz, México. Boletín de La Sociedad Botánica de México, 113, 107-113. Retrieved from http://www.scielo.org.mx/scielo.php? script $=$ sci_arttext\&pid=S0366-21282011000200006

Romero-Berny, E. I., Acosta-Velázquez, J., Tovilla-Hernández, C., Schmook, B., \& GómezOrtega, R. (2015). Cambios de cobertura y fragmentación de manglares en la región del Soconusco, Chiapas, México, 1994-2011. Revista Geográfica de América Central, 1(54), 153-169. https://doi.org/10.15359/rgac.1-54.7

Smith, T. ., Anderson, G. H., Balentine, K., Tiling, G., Ward, G. A., \& Whelan, K. R. T. (2009). Cumulative impacts of hurricanes on Florida mangrove ecosystems: Sediment deposition, storm surges and vegetation. Wetlands, 29, 24-34. https://doi.org/10.1672/08-40.1

Sokal, R., \& Rohlf, F. J. (1994). Biometry: The Principles and Practices of Statistical in Biological Research. (3rd ed.). San Francisco: Freeman Company.

Strauch, A. M., Cohen, S., \& Ellmore, G. S. (2012). Environmental Influences on the Distribution of Mangroves on Bahamas Island. Journal of Wetlands Ecology, 6, 16-24. https://doi.org/http://dx.doi.org/10.3126/jowe.v6i0.6081

Tapia-García, M., García-Abad, M. C., Carranza-Edwards, A., \& Vázquez-Gutiérrez, F. (2007). Environmental characterization of the continental shelf of the Gulf of Tehuantepec, Mexico. Geofisica Internacional, 46(4), 249-260. Retrieved from http://www.revistas.unam.mx/index.php/geofisica/article/view/40183

Thom, B. G. (1984). Coastal landforms and geomorphic processes. In S. C. Snedaker \& J. C. Snedaker (Eds.), The mangrove ecosystem: research method (1st ed., pp. 3-17). Bungay: UNESCO.

Torrescano, N., \& Islebe, G. A. (2006). Tropical forest and mangrove history from southeastern Mexico: a $5000 \mathrm{yr}$ pollen record and implications for sea level rise. Vegetation History and Archaeobotany, 15(3), 191-195. https://doi.org/10.1007/s00334-005-0007-9

Tovilla-Hernández, C., \& de la Lanza-Espino, G. (1999). Ecologia, Produccion y Aprovechamiento del Mangle Conocarpus erectus L., en Barra de Tecoanapa Guerrero, Mexico. Biotropica, 31(1), 121-134. https://doi.org/10.1111/j.17447429.1999.tb00123.x

Tovilla-Hernández, C., de la Lanza-Espino, G., \& Orihuela-Belmonte, D. E. (2001). Impact of logging on a mangrove swamp in South Mexico: Cost/benefit analysis. Revista de Biología Tropical, 49(2), 571-580. Retrieved from http://www.scielo.sa.cr/ scielo.php?script=sci_arttext\&pid=S0034-77442001000200015

Tovilla-Hernández, C., \& Romero-Berny, E. I. (2012). Diagnóstico estructural de los manglares de Chiapas y Oaxaca. In A. J. Sánchez, X. Chiappa-Carrara, \& R. Brito-Pérez (Eds.), Recursos acuáticos costeros del sureste. Volumen 1 (1st ed., pp. 257-279). Mérida: FOMIX-CONCITEY, RECORECOS, UNAM. Retrieved from http://www.sisal. unam.mx/recorecos/RED/RACS_files/RACS Vol I.pdf

Trejo-Torres, J., Durán, R., \& Olmsted, I. (1993). Manglares de la Península de Yucatán. In S. I. Salazar-Vallejo \& N. E. González (Eds.), Biodiversidad marina y costera de México (1st ed., pp. 661-672). México D.F.: CONABIO. 
Recibido:

$11 /$ febrero/2018

Aceptado: 10/diciembre/2018
Troche-Souza, C., Rodríguez-Zúñiga, M. T., Velázquez-Salazar, S., Valderrama-Landeros, L., Villeda-Chávez, E., Alcántara-Maya, A., ... Ressl, R. (2016). Manglares de México: extensión, distribución y monitoreo 1970/1980-2015 (1st ed.). México D.F.: CONABIO. Retrieved from http://bioteca.biodiversidad.gob.mx/janiumbin/detalle.pl?Id=20181030003622

Twilley, R. R. (1998). Mangrove Wetlands. In M. G. Messina \& W. H. Conner (Eds.), Southern Forested Wetlands: Ecology and Management (1st ed., pp. 445-473). Boca Raton: CRC Press LLC.

Upkong, I. E. (1995). An ordination study of mangrove swamp communities in West Africa. Vegetatio, 116, 147-159. Retrieved from https://www.jstor.org/stable/20046550

Urrego, L. E., Polanía, J., Buitrago, M. F., Cuartas, L. F., \& Lema, A. (2009). Distribution of Mangroves Along Environmental Gradients on San Andrés Island (Colombian Caribbean). Bulletin of Marine Science, 85(1), 27-43. Retrieved from http://www.ingentaconnect.com/contentone/umrsmas/bullmar/2009/00000085/000000 $01 / \operatorname{art} 00003$

Valdéz-Hernández, J. I. (2002). Aprovechamiento forestal de manglares en el estado de Nayarit, costa Pacífica de México. Madera y Bosques, 8(1), 129-145. Retrieved from http://myb.ojs.inecol.mx/index.php/myb/article/view/1296/1466

Valle, A., Osorno-Arango, A. M., \& Gil-Agudelo, D. L. (2011). Estructura y regeneración del bosque de manglar de la Ciénaga de Cholón, Isla Barú, Parque Nacional Natural Corales del Rosario y San Bernardo, Caribe Colombiano. Boletín de Investigaciones Marinas y Costeras, 40(1), 115-130. Retrieved from http://www.scielo.org.co/ $\mathrm{pdf} / \mathrm{mar} / \mathrm{v} 40 \mathrm{n} 1 / \mathrm{v} 40 \mathrm{n} 1 \mathrm{a} 07 . \mathrm{pdf}$

Villacencio-García, R., Espinoza-Aréchiga, J., Hernández-Álvarez, E., Gallegos-Rodríguez, A., \& Santiago-Pérez, A. L. (2009). Medición Forestal (1st ed.). Guadalajara: Amate.

Whiting, G. J., \& Chanton, J. P. (2001). Greenhouse carbon balance of wetlands: methane emission versus carbon sequestration. Tellus B: Chemical and Physical Meteorology, 53, 521-528. https://doi.org/10.3402/tellusb.v53i5.16628

Xiaonan, D., Xiaoke, W., Lu, F., \& Zhiyun, O. (2008). Primary evaluation of carbon sequestration potential of wetlands in China. Acta Ecologica Sinica, 28(2), 463-469. https://doi.org/10.1016/S1872-2032(08)60025-6

Zaldívar-Jiménez, M. A., Herrera-Silveira, J. A., Teutli-Hernández, C., Comín, F. A., Andrade, J. L., Coronado-Molina, C., \& Pérez-Ceballo, R. (2010). Conceptual Framework for Mangrove Restoration in the Yucatán Peninsula. Ecological Restoration, 28(3), 333342. https://doi.org/10.3368/er.28.3.333

Zarco-Espinosa, V. M., Valdéz-Hernández, J. I., Ángeles-Pérez, G., \& Castillo-Acosta, O. (2010). Estructura y diversidad de la vegetación arbórea del parque estatal Agua Blanca, Macuspana, Tabasco. Universidad y Ciencia, 26(1), 1-17. Retrieved from http://www.redalyc.org/articulo.oa?id=15416251001 